\title{
Inhomogeneous GaInNAs quantum wells: their properties and utilization for improving of p-i-n and p-n junction photodetectors
}

\author{
D. PUCICKI* \\ Faculty of Microsystem Electronics and Photonics, Wroclaw University of Science and Technolgy, \\ Janiszewskiego 11/17, 50-372 Wroclaw, Poland
}

\begin{abstract}
A theoretical study of electronic structures and optical properties of GaInNAs/GaAs quantum wells has been performed. The inhomogeneous distributions of indium and nitrogen atoms along the growth direction were discussed as the main factors having significant impact on the QWs absorption efficiency. The study was performed by applying the band anticrossing model combined with the envelope function formalism and based on the material parameters which can be found in the literature. Indeed, the electronic band structure of $15 \mathrm{~nm}$ thick uniform $\mathrm{Ga}_{0.7} \mathrm{In}_{0.3} \mathrm{~N}_{0.02} \mathrm{As}_{0.98} / \mathrm{GaAs} \mathrm{QW}$ was computed together with electronic structures of several types of inhomogeneous QWs, with the same total content of In and $\mathrm{N}$ atoms. It was found that presented inhomogeneities lead to significant quantum wells potential modifications and thus to spatial separation of the electrons and holes wave functions. On the other hand, these changes have a significant impact on the absorption coefficient behavior. This influence has been studied on the basis of simulated photoreflectance spectra, which probe the absorption transitions between QW energy subbands. The electronic structure of inhomogeneous QWs under the influence of electric field has also been studied. Two different senses of electric field vector (of $\mathrm{p}-\mathrm{i}-\mathrm{n}$ and $\mathrm{n}-\mathrm{i}-\mathrm{p}$ junctions) have been considered and thus, the improvement of such types of QWs-photodetectors based on inhomogeneous GaInNAs QWs has been proposed.
\end{abstract}

Keywords: dilute nitrides; quantum well; structural inhomogeneities; photodetectors

\section{Introduction}

In and $\mathrm{N}$ containing $\mathrm{GaAs}$ bulk and related quantum wells (QWs) were considered as potential candidates in optoelectronic devices applications, e.g. in solar cells [1], photodetectors [2, 3] and lasers $[4,5]$, also for optical fiber communications. The unusual influence of nitrogen on the electronic band structure of GaInNAs alloys, essentially the large band gap reduction $[6,7]$ results in a wide range of applications of such material. Over the last few years, the growth of GaInNAs alloys has been demonstrated by both metalorganic vapor phase epitaxy (MOVPE) and molecular beam epitaxy (MBE), and significant improvement of GaInNAs optical and structural quality was achieved [4, 5, 8]. Moreover, these alloys can ensure the possibility of matching to several substrates (e.g. GaAs

*E-mail: Damian.Pucicki@pwr.edu.pl or InP), which are commonly used in the optoelectronic devices technology. However, in case of more advanced devices, engineering of carrier confinement potential, densities of states and carrier dynamics forced the use of unmatched GaInNAs layers in a form of quantum wells. In addition, more complex alloys, like GaInNAsSb, are also considered for longer emission and devices improvement [9, 10]. However, the increase of $\mathrm{N}$ content in GaInNAs epitaxial layers requires much lower growth temperatures and significantly higher concentrations of the nitrogen source material in epitaxial reactors $[8,11]$. Such growth conditions cause strong degradation of their optical properties $[12,13]$. The deterioration of crystalline quality of low-temperature crystallized GaInNAs layers can be partially reversed by means of rapid thermal annealing (RTA) or optical annealing (OA) [8, 9]. The nitrogen related point defects, which are results of strong growth condition modifications, are often mentioned as a main 
reason of material quality deterioration. Moreover, in the literature, it can be found that the metastability of GaInNAs alloys leads to strong phase segregation [14-16] and interdiffusion [17, 18]. However, not many attempts in calculating the influence of composition inhomogeneity on the optical transitions in dilute nitride based systems are given in the literature [18]. Consideration of these types of structural changes, especially in case of quantum wells layers, can be a key point in the optoelectronic devices improvement. Therefore, it is really important to undertake all possible measures to improve absorption characteristics of optoelectronic devices, what was also a motivation for the analysis of the influence of structural inhomogeneities on the properties of GaInNAs/GaAs quantum wells.

\section{Theoretical approach}

The calculations of QWs energy band structure were performed within the framework of the usual envelope function approximation and with neglecting excitonic effect $[19,20]$. In the performed calculation, relative position of bands in heterostructures was based on experimentally obtained parameters representing absolute valence band position as well as on the calculated value of band gap of each material. All necessary parameters of multicomponent alloys were estimated using simple formula for linear approximation (Vegard law) [21]. The change of the band gap of InGaAs alloy was calculated according to a nonlinear approximation considering the bowing parameter. The band anticrossing model was applied in order to calculate the changes of the conduction band of GaInNAs due to the presence of nitrogen atoms in the alloy [22]. The effect of electron effective mass increase for nitrogen containing materials has also been calculated in accordance with BAC model. According to that model it was assumed that the influence of nitrogen localized states on the valence band structure is neglected and the effective mass of the light and heavy holes does not change due to the presence of nitrogen atoms in the crystal. Nevertheless, the biaxial strain was calculated based on the Pikus-Bir Hamiltonian and the energy shifts and splitting of the valence band into heavy and light holes, caused by hydrostatic and shear stresses, were also considered. The necessary material parameters, such as valence band energy, elastic stiffness constants, deformation potentials, lattice parameters, nitrogen and host matrix energies and interaction parameters as well as bowing parameters, were taken from the literature $[21,23]$. The numerical matrix methods and Sturm-Martin-Dean algorithm were applied in order to solve Schrödinger equation and to calculate eigenvalues and eigenvectors $[24,25]$. More detailed information about utilized band structures calculations can be found in the author's earlier work [16].

\section{QWs composition profiles}

In order to investigate different types of inhomogeneities of the GaInNAs QWs on their properties, several profiles of indium and nitrogen distributions along the growth direction of the wells have been considered. However, the exact comparative analysis of electronic and optical properties of the GaInNAs/GaAs quantum wells requires specifying the common features of analyzed structures. For this purpose, the total indium and nitrogen contents in all considered QWs have been set constant. It means, that in case of all considered structures, the values of integrals, which represent the surface area under the In or $\mathrm{N}$ compositions profiles, are equal. This assumption allows one to investigate only the influence of the composition profiles changes on the QWs properties. All assumed profiles of composition were presented in Fig. 1. As a reference structure "Uniform" $\mathrm{Ga}_{0.7} \mathrm{In}_{0.3} \mathrm{~N}_{0.02} \mathrm{As}_{0.98} / \mathrm{GaAs}$ quantum well has been considered (Fig. 1a). The other constructions of the structures with inhomogeneous QWs (Fig. 1b to Fig. 1e) have been assigned as a "Error QW", "Linear QW", "Exponential QW" and "Real QW". In case of "Error QW" the only interdiffusions of In and $\mathrm{N}$ atoms have been considered, which resulted in small QW broadening. The diffusion lengths of $0.75 \mathrm{~nm}$ for nitrogen and indium have been assumed the same. The changes in composition profiles at the interfaces (an increase/decrease of the In and $\mathrm{N}$ amount) are described in this case by error 
function. Furthermore, the interdiffiusion-induced changes of the in-plane strain on electrons and holes confinement profiles have also been taken into account in this structure. In case of next two QWs constructions, "Linear QW" and "Exponential QW", the only monotonic linear and exponential changes of In and $\mathrm{N}$ contents have been assumed. Moreover, the inverse slops have been assumed for indium and nitrogen composition profiles, what is in agreement with the observed phase segregation in the real structures and change of nitrogen incorporation efficiency on the indium content. The last assumed construction of the GaInNAs QW considers the interdiffusion of atoms at the interfaces of the well, phase segregation and dependence of the nitrogen incorporation efficiency on the indium content, as well. In this case, the same assumptions have been adopted for interface interdiffusion of the In and $\mathrm{N}$ as for "Error QW" structure. Such construction is called "Real QW". In addition, the InGaAs QW with linear change of composition has also been computed to present the significantly different impact of alloy inhomogeneities on the QWs made of ternary materials.

\section{Electronic structure analysis}

The assumed profiles of compositions of GaInNAs quantum well layers were used in order to calculate the QWs potential distribution along their characteristic direction (corresponding to the growth direction of the real epitaxial structures). Afterwards, the eigenvalues and eigenvectors of the wells were computed. As results of these calculations, complete electronic band structures of considered GaInNAs/GaAs quantum wells have been achieved (Fig. 2).

On the basis of the graphical presentation of electronic band structures of the GaInNA/GaAs quantum wells, which differ in In and $\mathrm{N}$ composition profiles, one can conclude that the considered inhomogeneities lead not only to the QWs potential modification, but most of all to the change of the electrons and holes energy states (colored blue and red in Fig. 2, respectively) and corresponding wave functions shapes. Furthermore, the completely different impact of indium and nitrogen on the band structure of GaInNAs alloy is the reason of huge spatial separation of the wave functions of electrons and holes. Thus, strong influence of these structural inhomogeneities on the QWs optoelectronic properties could be expected. Such behavior is in a big contrast to the inhomogeneous quantum wells made of ternary alloys, such as InGaAs or AlGaAs. In case of such simple QWs, the impact of indium content perturbation has a small influence on quantum well density of states (DOS) and energy states but does not lead to strong spatial separation of electron and holes wave functions (Fig. 3). The study of graded AlGaAs quantum well can be found in the literature [26].

The more detailed data obtained from the quantum wells band structure calculations are presented in Table 1. The adopted accuracy of the presented data results from the accuracy of the photoreflectance (PR) measurements and accuracy of the PR spectra analysis, from which the energies and amplitudes of the resonances, can be estimated with the accuracy of $\sim 1 \mathrm{meV}$ and $\sim 1 \%$, respectively. For each considered GaInNAs/GaAs quantum well, the energies $E$ of three main transitions between electron and heavy holes states - $(1 \mathrm{e} 1 \mathrm{hh}$, $2 \mathrm{e} 2 \mathrm{hh}$ and $3 \mathrm{e} 3 \mathrm{hh}$ ) and corresponding wave functions overlap integrals $\left\langle\Psi_{\mathrm{e}} \Psi_{\mathrm{hh}}\right\rangle$ were collected. One can see that despite the constant total amount of In and N in the discussed GaInNAs/GaAs QWs, strong change of ground state transitions energy can be observed. The effect of increased depth of potential wells in a part of the inhomogeneous quantum wells is partially eliminated by narrowing the effective thickness confining the carriers, similar to that described in case of quantum-confined Stark effect (QCSE). Nevertheless, the ground state transition energies vary in the range of $1.037 \mathrm{eV}$ to $1.116 \mathrm{eV}$ for the Exponential QW and Error QW, respectively. Moreover, wave function overlap integrals also vary from 0.143 to 0.998 for the Exponential QW and Uniform QW, respectively.

\section{Optical properties analysis}

Since the electron transition probability is dependent, among the others, on the value of wave functions overlap, according to Fermi's golden 


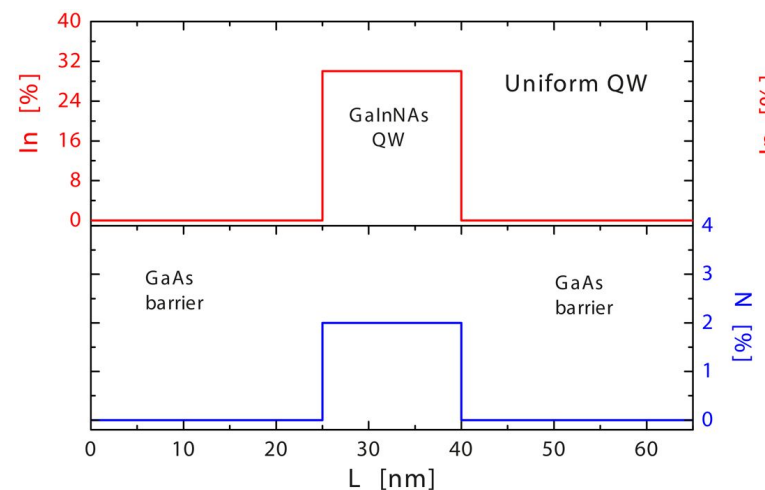

(a)

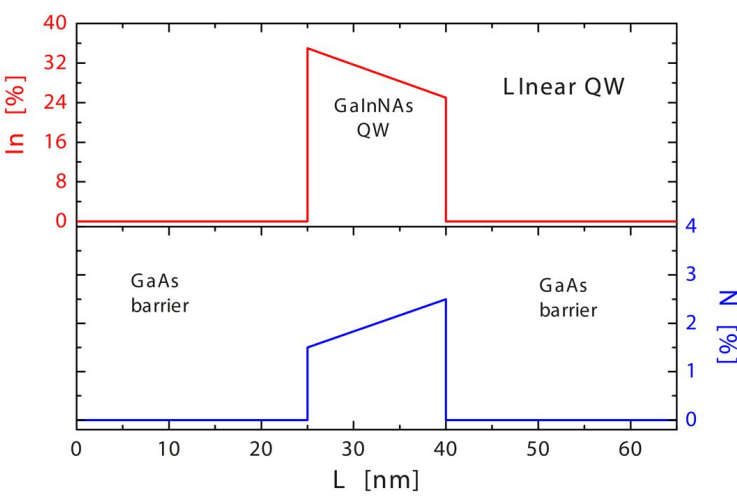

(c)

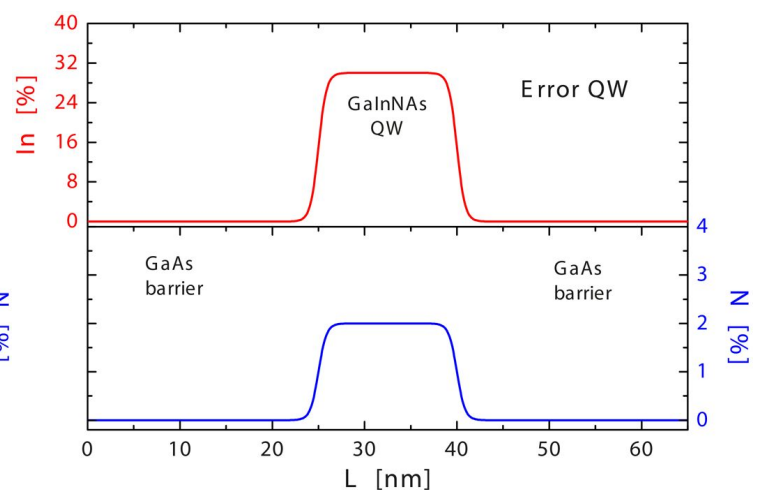

(b)

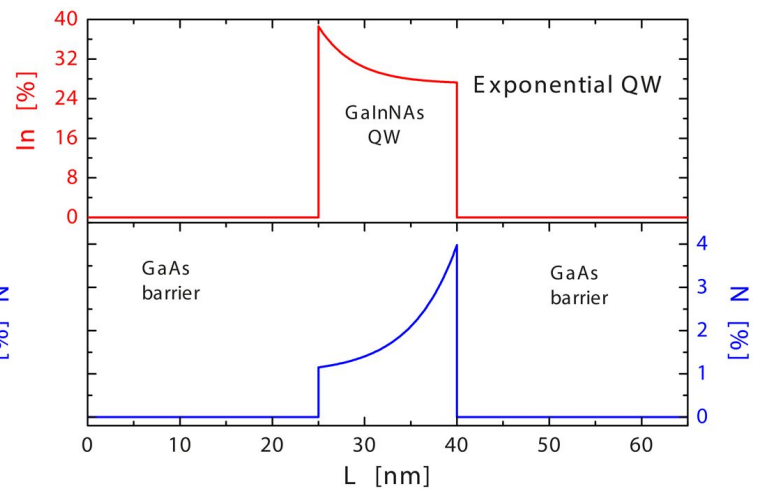

(d)

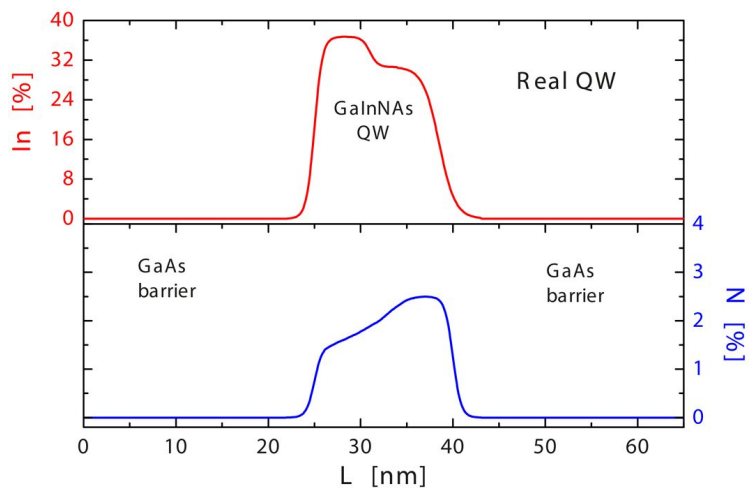

(e)

Fig. 1. Profiles of In and $\mathrm{N}$ concentration along the GaInNAs quantum wells layers with the assumed types of structural inhomogeneities: (a) Uniform QW, (b) Error QW, (c) Linear QW, (d) Exponential QW, (e) Real QW.

rule, the optical transitions probabilities should also be modified. The best method for probing the energies and the strengths of quantum wells optical transitions is the modulated reflectance spectroscopy [27]. As results of such measurements, the spectrum of resonance is obtained. The shape of that spectrum depends mainly on the transitions probabilities - amplitudes of resonances, energies of electron transitions - positions of the resonances as well as on the broadening of the quantum well subbands, broadening and overlapping of the resonances.

To study the impact of structural inhomogeneities of GaInNAs quantum wells on the 


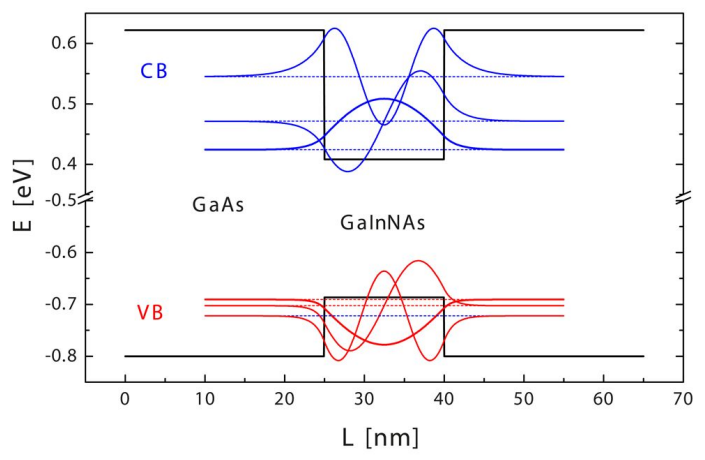

(a)



(c)

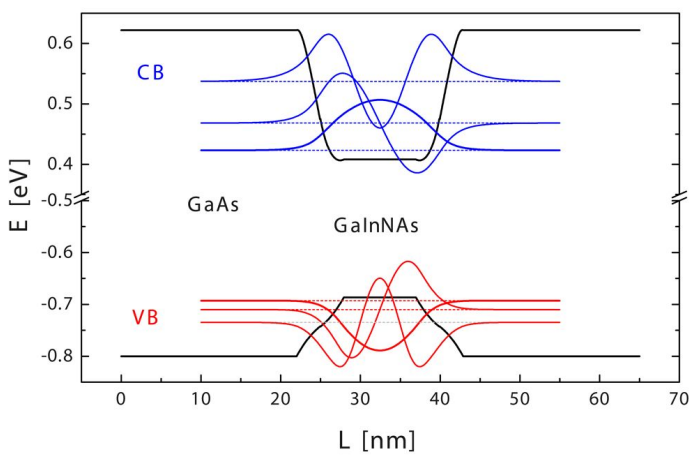

(b)

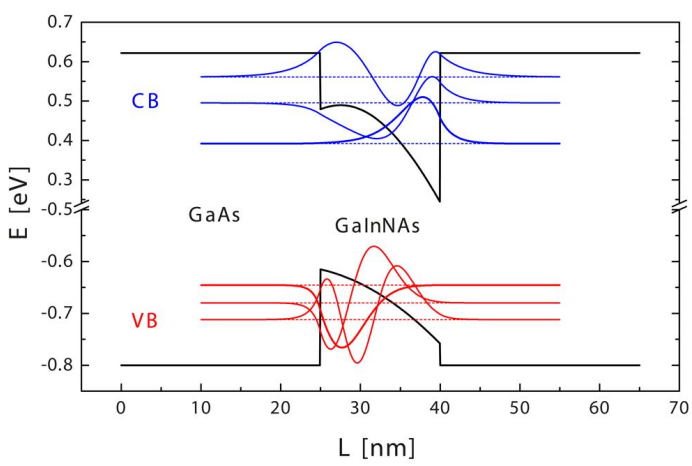

(d)

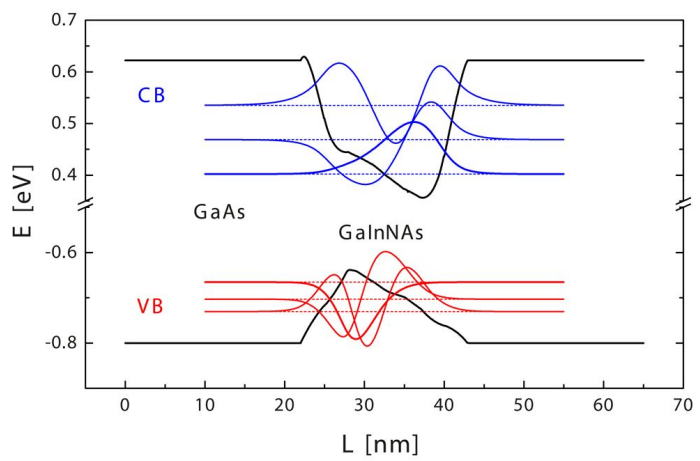

(e)

Fig. 2. Electronic band structures of GaInNAs/GaAs quantum wells with different In and $\mathrm{N}$ composition profiles: (a) Uniform QW, (b) Error QW, (c) Linear QW, (d) Exponential QW, (e) Real QW. Electrons and heavy holes energy states and wave functions are colored blue and red, respectively.

optical properties, the photoreflectance spectra have been simulated. As initial parameters for that analysis, the values of energies and wave functions overlap (Table 1) obtained from the previous calculations were applied. For all of the considered
GaInNAs/GaAs inhomogeneous QWs, the energies of the individual electron transitions and wave functions overlap integrals were assigned to the subsequent simulated resonances. As parameters influencing 
Table 1. Energies of QWs electron transitions $E$ and corresponding wave function overlap integrals $\left\langle\Psi_{\mathrm{e}} \Psi_{\mathrm{hh}}\right\rangle$ describing the electronic band structures of GaInNAs/GaAs quantum wells.

\begin{tabular}{ccccccc}
\hline $\begin{array}{c}\text { Electron transition type } \\
\text { Type of QW }\end{array}$ & \multicolumn{2}{c}{$1 \mathrm{e} 1 \mathrm{hh}$} & \multicolumn{2}{c}{$2 \mathrm{e} 2 \mathrm{hh}$} & \multicolumn{2}{c}{$3 \mathrm{e} 3 \mathrm{hh}$} \\
$\mathrm{E}]$ & $\left\langle\Psi_{1 \mathrm{e}} \Psi_{1 \mathrm{hh}}\right\rangle$ & $\mathrm{E}[\mathrm{eV}]$ & $\left\langle\Psi_{2 \mathrm{e}} \Psi_{2 \mathrm{hh}}\right\rangle$ & $\mathrm{E}[\mathrm{eV}]$ & $\left\langle\Psi_{3 \mathrm{e}} \Psi_{3 \mathrm{hh}}\right\rangle$ \\
\hline \hline Uniform & 1.115 & 0.998 & 1.174 & 0.989 & 1.267 & 0.963 \\
Error & 1.116 & 0.978 & 1.178 & 0.947 & 1.272 & 0.901 \\
Linear & 1.090 & 0.450 & 1.175 & 0.009 & 1.271 & 0.249 \\
Exponential & 1.037 & 0.143 & 1.175 & 0.470 & 1.273 & 0.239 \\
Real & 1.068 & 0.414 & 1.172 & 0.003 & 1.266 & 0.160 \\
\hline
\end{tabular}

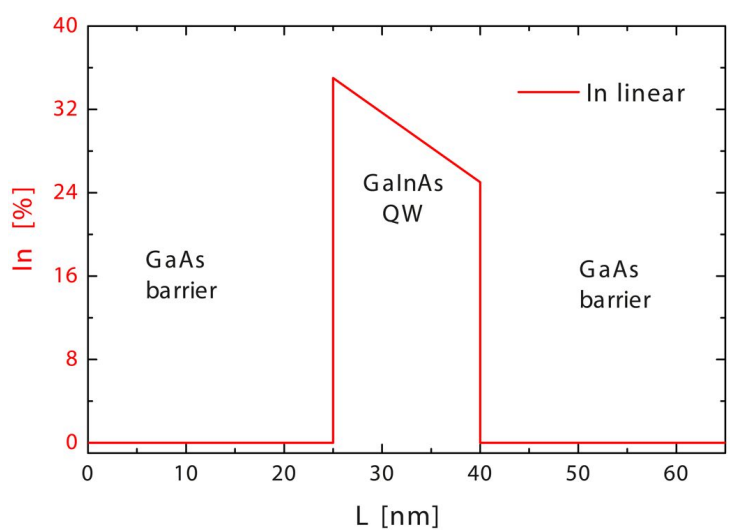

(a)

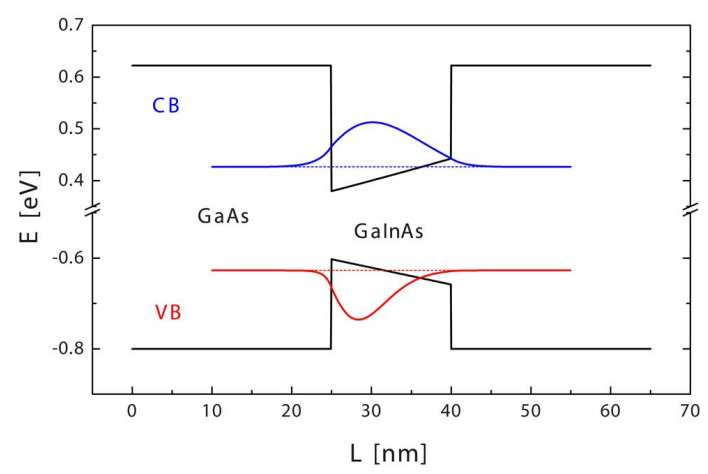

(b)

Fig. 3. Structural and band structure data describing inhomogeneous InGaAs/GaAs quantum well with linear change of indium content: (a) profile of In concentration along the QW thickness, (b) electronic band structures.

the amplitudes of the simulated resonances, the squares of the modules $\left|\left\langle\Psi_{\mathrm{ne}} \Psi_{\mathrm{mhh}}\right\rangle\right|^{2}$ were assumed. The broadening of the resonances accepted for the simulation was based on the values obtained earlier from the experiment and analysis of measured spectra [16]. The broadening simulates the influence of planar thickness and composition inhomogeneities of the QWs. Significant differences in optical properties of the inhomogeneous quantum wells (Fig. 4) can be seen from performed analysis. However, each type of assumed heterogeneity of GaInNAs/GaAs QWs leads to a deterioration in the optical properties of these structures and attenuation of the resonances in comparison to a homogeneous $\mathrm{Ga}_{0.7} \mathrm{In}_{0.3} \mathrm{~N}_{0.02} \mathrm{As}_{0.98} / \mathrm{GaAs} \mathrm{QW}$ quantum well.

\section{Photodetectors improvement}

Grading of alloy composition is a common technique in designing optoelectronic devices. In case of graded index separate confinement heterostructure (GRINSCH) the composition grading is applied to cladding layers in order to improve waveguiding, thus, lasing threshold and beam quality [28]. The quantum well is usually not graded in such emitting devices. Inhomogeneous quantum wells have been of recent interest since it is well known that in GaN-based MQW structures, piezoelectrical surface charge deforms quantum well potential. However, in case of a GaAs-based p-n or p-i-n photodiodes the junction built-in electric field has also significant influence on the quantum well electronic band structure and its absorption efficiency [29]. In addition, the influence is enhanced due to reverse bias applied in a standard working condition of that type of semiconductor photodetectors. The electromodulation effect and adverse impact of the electric field on the quantum wells 


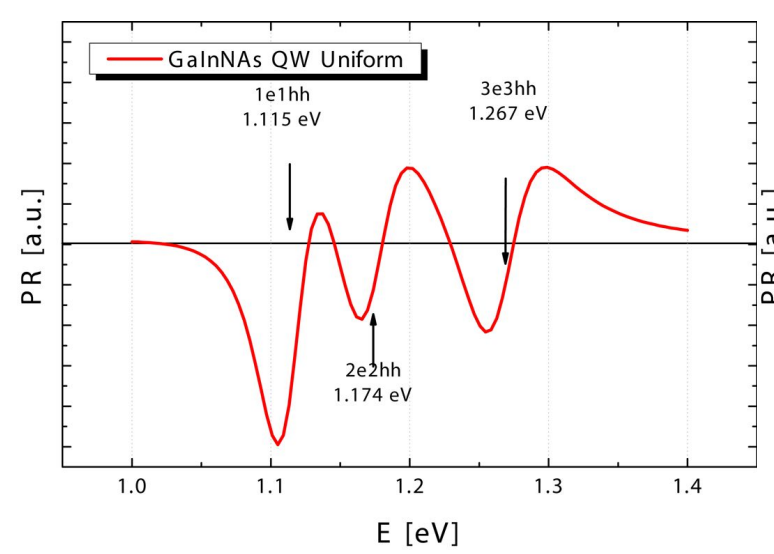

(a)

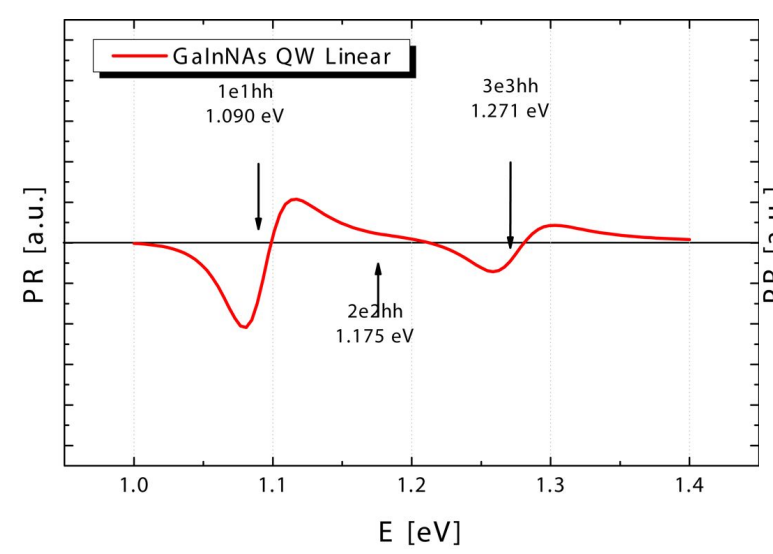

(c)

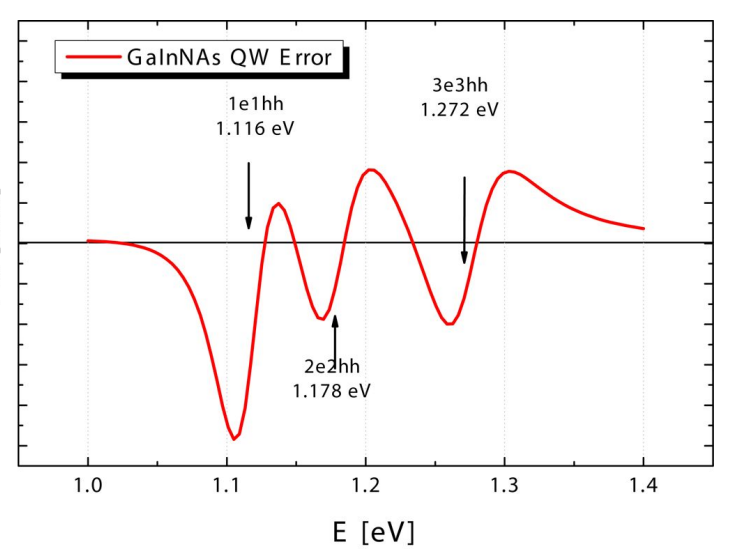

(b)



(d)

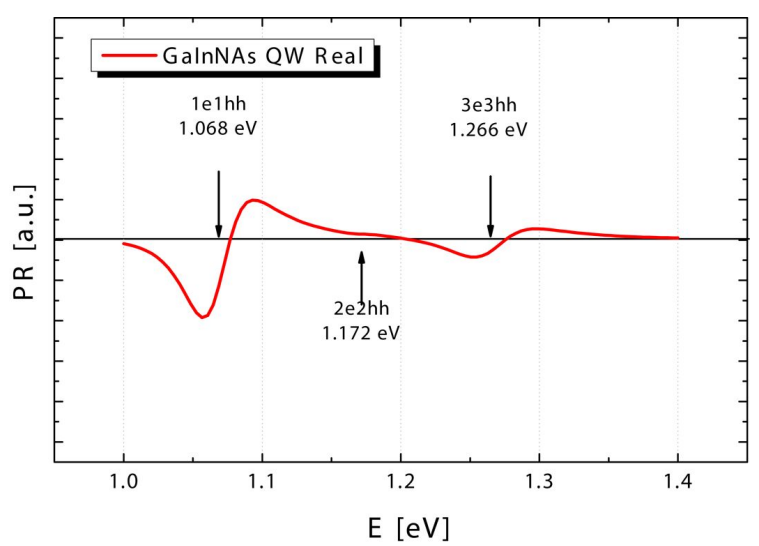

(e)

Fig. 4. Photoreflectance spectra of GaInNAs/GaAs quantum wells with different In and N composition profiles: (a) Uniform QW, (b) Error QW, (c) Linear QW, (d) Exponential QW, (e) Real QW.

is described by well-known QCSE. Since inhomogeneous GaInNAs/GaAs QWs can ensure an initial (without any electric field acting on the well) spatial separation of electrons and holes wave functions, not only in case of considered in this work structures but also in the real structures [30], one 
may wish to use that effect to reduce the impact of built-in electric field of the junction on attenuation of absorption efficiency of the QWs, thus, on deterioration of photodetectors absorption characteristics to reduce the adverse impact of Stark effect on absorption efficiency.

Table 2. Values of wave functions overlap integrals corresponding to ground state transitions of a step-like GaInNAs/GaAs QW under no electric field and under influence of electric fields with the opposite sense.

\begin{tabular}{cccc}
\hline & p-i-n & & n-i-p \\
\hline \hline $\mathrm{E}[\mathrm{kV} / \mathrm{cm}]$ & 31.7 & 0.0 & 31.7 \\
$\left\langle\Psi_{1 \mathrm{e}} \Psi_{1 \mathrm{hh}}\right\rangle$ & 0.7728 & 0.3986 & 0.2436 \\
$\left|\left\langle\Psi_{1 \mathrm{e}} \Psi_{1 \mathrm{hh}}\right\rangle\right|^{2}$ & 0.597 & 0.1589 & 0.05934 \\
\hline
\end{tabular}

To confirm these possibilities, a theoretical study of an electric field impact on the inhomogenous QW was conducted. As an example, the band structure of the real inhomogeneous GaInNAs/GaAs step-like quantum well was taken under consideration. Presented kind of quantum well layer inhomogeneity was achieved during an experiment of MOVPE growth. The detailed information about the growth condition, characterization and structural data of that sample can be found in the literature [30]. Such step-like QW was considered as an absorber of the $\mathrm{p}-\mathrm{i}-\mathrm{n}$ and $\mathrm{n}-\mathrm{i}-\mathrm{p}$ photodetector structures which differ in electric field sense which affects the quantum wells located in the center of the junctions. In Fig. 5, electronic band structures of inhomogeneous step-like GaInNAs/GaAs quantum well are presented under different conditions of electric field. One can see that without electric field the conduction band ground state wave function is shifted left, towards nitrogen rich sublayer of the QW. The adequate valence band wave function is shifted right, towards indium rich QW sublayer (Fig. 5a). Such spatial separation is enhanced when QW is placed/located in n-i-p junction of photodetector (Fig. 5b) and reduced in opposite p-i-n structure of the device (Fig. 5c). Furthermore, the wave function distribution can be additionally tuned, when reverse bias of the considered junctions is applied. In first case of n-i-p photodetector

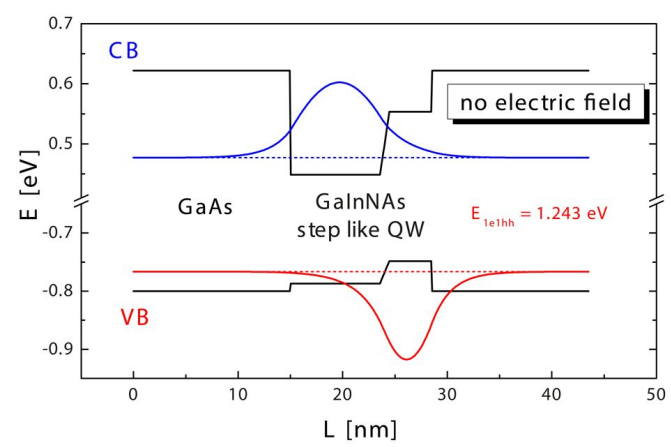

(a)

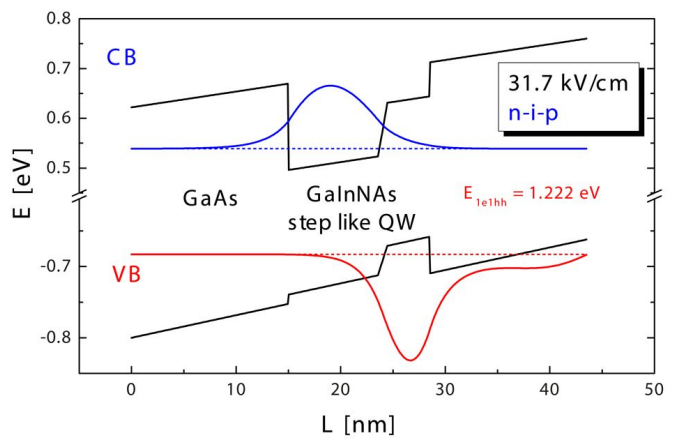

(b)

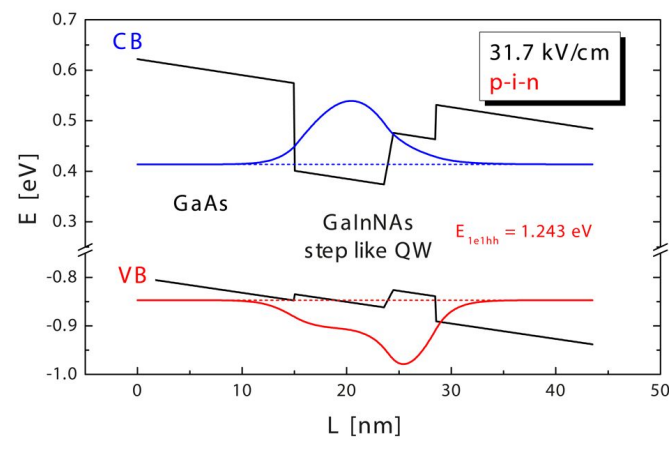

(c)

Fig. 5. Electronic band structures of real inhomogeneous step-like GaInNAs/GaAs quantum well: (a) with no electric field acting on the energy bands, (b) and (c) under the influence of electric field of $31.7 \mathrm{kV} / \mathrm{cm}$ of a n-i-p and p-i-n junction, respectively.

structure, the reverse bias will cause stronger wave functions separation and, consequently, will deteriorate the absorption efficiency. In case of inverse $p-i-n$ structure, the reverse bias will result in better 
overlapping of the wave functions making the absorption more efficient. The values of the ground states wave functions overlaps integrals, which are results of carried out calculations, are summarized in Table 2. Furthermore, the squares of modules of that parameter are also presented in order to emphasize the difference in the value of the factor taken into account in Fermi's golden rule equation and influencing the probability of absorption.

\section{Conclusions}

In this work, compositionally graded, inhomogeneous GaInNAs/GaAs quantum wells were discussed. The influence of several types of inhomogeneities of these QWs on their electronic band structure, thus, on their optical properties, was studied. It was shown that the interdiffusion and phase segregation occurring in the QW layers made of epitaxialy grown metastable materials lead to significant attenuation of QWs absorption. Thus, these structural changes must be taken into account when the device structures are designed. Notwithstanding, the discussed defects of GaInNAs-based quantum wells, which were considered to be the reason of optical properties deterioration, can be utilized in $\mathrm{p}-\mathrm{n}$ and $\mathrm{p}-\mathrm{i}-\mathrm{n}$ photodetectors to improve their quantum efficiency. From the point of view of thermodynamic processes, which rule the epitaxial growth and phase segregation, it is often impossible to freely change the directions of indium and nitrogen changes within the quantum well layers in respect to their growth direction. This is the reason, why the change of the electric field sense, which can be inverted by utilization of $p-i-n$ and $n-\mathrm{i}-\mathrm{p}$ junctions, is proposed to fabricate the photodetector and to compensate the adverse impact of QCSE on the absorption efficiency.

\section{References}

[1] Langer F., Perl S., Höfling S., Kamp M., Appl. Phys. Lett., 106 (2015), 233902.

[2] Balkan N., Erol A., Sarcan F., Al-Ghuraibawi L.F.F., Nordin M.S., Superlattice Microst., 86 (2015), 467.

[3] Sarcan F., Nordin M.S., Kuruoglu F., Erol A., VICKERS A.J., Superlattice Microst., 102 (2017), 27.
[4] Bisping D., Höfling S, Pucicki D., Fischer M., FORCHEL A., Electron. Lett., 44 (2008), 737.

[5] Bisping D., PuCicki D., Höfling S., HaberMANN S., EWERT D., Fischer M., Koeth J., ForCHEL A., IEEE Photonic Tech. L., 20 (2008), 1766.

[6] Vurgaftman I., Meyer J.R., J. Appl. Phys., 94 (2003), 3675.

[7] Tixier S., Webster S.E., Young E.C., Tiedje T., Francoeur S., Mascarenhas A., Wei P., SchiETtekatte F., Appl. Phys. Lett., 86 (2005), 112113.

[8] Bisping D., Pucicki D., Fischer M., Höfling S., Forchel A., J. Cryst. Growth, 311 (2009), 1715.

[9] Baranowski M., Kudrawiec R., SypereK M., Misiewicz J., SARMiento T., Harris J.S., Nanoscale Res. Lett., 9 (2014).

[10] Bank S.R., Bae H.P., Yuen H.B., Wistey M.A., GodDARD L.L., HARRIS JR. J.S., Electron. Lett., 42 (2006).

[11] Ściana B., Pucicki D., Radziewicz D., SeraFIŃCZUK J., KOZŁOWSKI J., PASZKIEWICZ B., TŁaczaŁa M., PoloczeK P., SĘK G., MisiewicZ J., Vacuum, 82 (2008), 377.

[12] Baranowski M., Kudrawiec R., Misiewicz J., Hammar M., Appl. Phys. A-Mater., 118 (2015), 479.

[13] Pan Z., Li L.-H., DU Y., Lin Y.-W., Wu R.-H., Chinese Phys. Lett., 18 (2001), 659.

[14] Luna E., Trampert A., Pavelescu E.-M., Pessa M., New J. Phys., 9 (2007), 1.

[15] LiU H.F., Xiang N., Chua S.J., Appl. Phys. Lett., 89 (2006), 071905.

[16] Pucicki D., Bielak K., Ściana B., RADZIEWICZ D., LATKOWSKA-BARANOWSKA M., Kováč J., Vincze A., TŁaczaŁA M., J. Cryst. Growth, 433 (2016), 105.

[17] Chan M.C.Y., Surya Ch., Wai P.K.A., J. Appl. Phys., 90 (2001), 197.

[18] Ryczko K., Sęk G., Misiewicz J., Superlattice Microst., 37 (2005), 273.

[19] BurT M.G., Semicond. Sci. Tech., 3 (1988).

[20] Sun Y., Thompson S.E., Nishida T., Strain Effect in Semiconductors. Theory and Device Application, Springer Science \& Business Media, New York, 2009.

[21] MeI T., J. Appl. Phys., 101 (2007), 013520.

[22] Shan W., Walukiewicz W., Ager J.W., Haller E.E., Geisz J.F., Friedman D.J., Olson J.M., Kurtz S.R., Phys. Rev. Lett., 82 (1999), 1221.

[23] Vurgaftman I., Meyer J.R., Ram-Mohan R., J. Appl. Phys., 89 (2001), 5815.

[24] Salejda W., Just M., TyC H., CMST, 6 (2000).

[25] Salejda W., TyC H., Just M., Algebraiczne metody rozwiazywania równania Schrödingera, Wydawnictwo Naukowe PWN, Warszawa, 2002. (in Polish).

[26] Li Z.S., MENSZ P.M., Numerical simulation of composition grading in active layer of quantum well lasers, in: Wun̈sche H.-J., PIPREK J., BANDElOW U., WENZEL H. (Eds.), NUSOD '05. $5^{\text {th }}$ International Conference on Numerical Simulation of Optoelectronic Devices 2005, Piscataway, New Jersey, 2001, p. 77. 
[27] Misiewicz J., Kudrawiec R., Opto-Electron. Rev., [30] Pucicki D., Bielak K., Badura M., Dawid20 (2012), 101.

OWSKI W., ŚCIANA B., Microelectron. Eng., 161 (2016), 13.

[28] Tsang W.T., Appl. Phys. Lett., 40 (1982), 217.

[29] Lordi V., YUEN H.B., BANK S.R., HARris J.S., Appl. Phys. Lett., 85 (2004), 902.

Received 2017-10-23 Accepted 2017-12-12 